\title{
Performance Evaluation of Low Cost Analog Feedback Active Noise Cancellation System
}

\author{
Soo Joon Yee, Pang Wai Leong, Chan Kah Yoong
}

\begin{abstract}
Acoustic noise can be reduced by active noise cancellation (ANC) and passive noise cancellation (PNC) algorithm. The PNC can effectively attenuate the noise with high frequency, but not the noise with low frequency. ANC is one of the promising solution to reduce the low frequency noise. Commercial ANC headphones often use the digital signal processor (DSP) to perform the noise cancellation algorithm to cancel the annoying acoustic noise, but the cost is relatively high. A low-cost ANC solution is urgently needed to reduce the acoustic noise. The relationship between the frequency, distance and degree of magnitude of the noise level are also evaluated in this paper.
\end{abstract}

Index Terms: Active noise cancellation, Analog feedback.

\section{INTRODUCTION}

Noise (undesired sound) causes disturbance and disruption. The audio noise or excessive noise can be harmful to one's hearing like engines, blower and aircraft. People nowadays tend to listen music using their audio headset during the journey on traveling and working as the headset is affordable since the advances of electronics. However, the daily activities and transportations such as airplane and train are often noisy. When the noise level is high, it leads to uncomfortable experience, and might cause ear fatigue. Besides, there is a study found that human will encounter physiological, psychological and physical effects due to the exposure to high level of noise [1].

The common solution to solve this issue is by the passive noise cancellation that uses barriers, silencers, barriers and absorption materials that can block the sound transmission without using any electronics circuit. However, this technique can only attenuate the noise that is medium and high frequencies and ineffective on the noise signal with low frequency. Hence, the active noise cancellation is introduced as it is a much more effective solution in attenuates and cancels the low frequency noise. This concept was first appeared in Paul Lueg's patent "Process of silencing sound oscillations" in 1936 [2]. He described the patent by inverting the polarity and decaying the phase of the wave to cancel the sinusoidal tones in ducts. The ANC system generates an opposite noise signal to cancel the existing noise signal. The ANC system is operated according to the superposition theory. The ANC system generates a $180^{\circ}$ opposite phase, same

Revised Manuscript Received on August 18, 2019.

Soo Joon Yee, Faculty of Engineering, Multimedia University, Cyberjaya, Malaysia.

Pang Wai Leong, Faculty of Engineering, Multimedia University, Cyberjaya, Malaysia.

Chan Kah Yoong, Faculty of Engineering, Multimedia University, Cyberjaya, Malaysia. amplitude anti-noise signal and combined it with the primary noise signal, in order to cancel both noise signals through destructive interference.

There is a wide range of literature about the active noise cancellation and there are different algorithms and approaches to design the ANC circuit. The common ANC configuration from previous works is based on the Mean Square Error Minimization to simplify the computation of the propagation delay. Filtered-X Least Mean Square is proposed for ANC [3]. Besides, a variety of algorithms are proposed which are based on genetic networks, Recursive least square filter, Neural networks, Kalman filter, and Lattice filter [4]-[6].

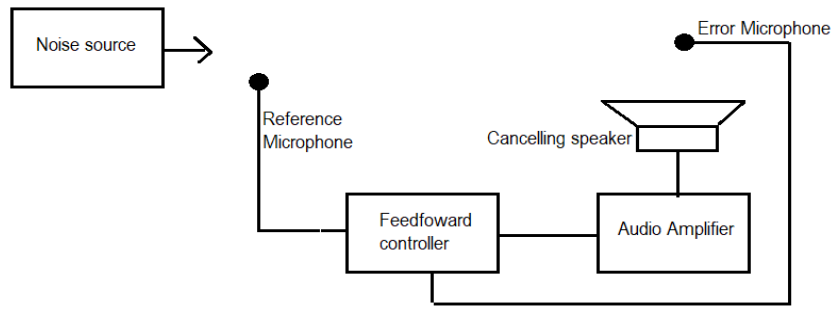

Figure 1. Feedforward ANC System.

There are two different types of controllers which are the feedforward controller and the feedback controller that used in the existing ANC system. A feedforward controller, a reference microphone, a speaker, an error microphone and an audio amplifier are needed in a feedforward ANC system (Figure 1). The error microphone captures the noise signal while the reference microphone captures the residual signal. The reference microphone hears the noise before the listener does. It then processes the noise picked up, generates the anti-noise signal and forwarded this signal to the cancelling speaker. The microphone can receive the noise earlier and hence having more interval to respond and generate the inverted waves. However, this approach cannot calibrate the signal as it never picks up the anti-noise it generates.

The feedback ANC system consists of a feedback controller, an error microphone, an audio amplifier and a speaker (Figure 2). The error microphone captured the noise signal, then inverted and the feedback signal to the cancelling speaker. In this approach, the error microphone is close to the listener and located in front of the cancelling speaker, so it is able to pick up the resulting signal the same way as the listener does. Since it picks up on what the listener hears, the feedback ANC has higher efficiency to improve various kinds of signal frequencies. Besides, the feedback ANC also supports a broader range of frequencies. The feedforward system has a 
better attenuation of noise compared to the feedback system. However, the feedforward system is costlier as it uses two microphones compared to the feedback system which uses only one.

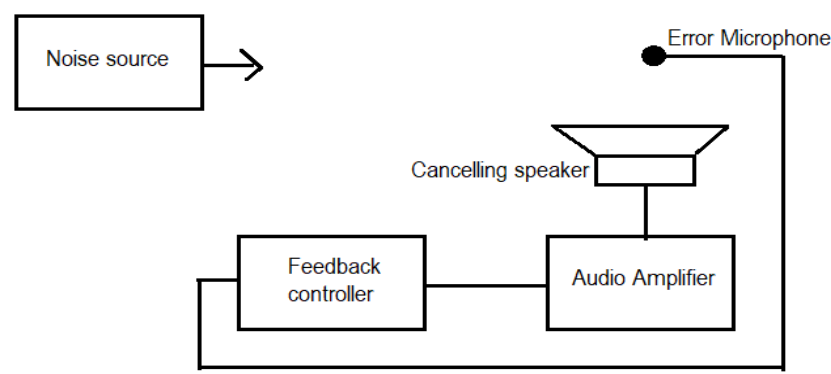

Figure 2. Feedback ANC System.

The commercial ANC circuits are commonly using the floating-point processing-type digital signal processor (DSP) as the core. The consequence is that the cost of the product will be relatively high. In this work, a low-cost analog feedback ANC system is designed using the basic electronics components.

\section{II.ANALOG FEEDBACK ANC}

The circuit proposed can be separated into four major stages which are DC filter, pre-amplifier, all pass filter and summing amplifier (Figure 3). The DC filter is used to block the DC bias settings from AC signal and also to reduce the parasitic capacitive coupling. In Figure 4, the capacitor and resistor at the left top acts as a low pass filter. The signal with a frequency lower than the cutoff frequency will be passed the filter, or else the signal will be attenuated.

This is necessary as high frequency noise may cause aliasing to the input signal which will potentially lead to indistinguishable of the signals and difficulty of the circuitry to identify the frequency of the signal. Besides, DC offset is removed by the capacitor and only the noise signal detected by the microphone is able to pass through.

The output of the DC Filter is forwarded to the pre-amplifier. The non-inverting operational amplifier circuit amplifies the incoming signal. The pre-amplifier amplified the weak signal captured by the microphone in order to increase the signal amplitude so that it can be processed. The gain of the pre-amplifier is controlled by the ratio of the resistors $\mathrm{R} 7$ and $\mathrm{R} 8$.

At room temperature, the speed of the sound is $343 \mathrm{~m} / \mathrm{s}$. However, the electrical signal operates at the speed of light. This means that the speed of the electrical signal passing through the circuit is much faster than the noise from the environment which travel at the speed of sound. In a nutshell, the anti-noise generated by the speaker will arrive earlier than the noise from the noise source which increase the difficulty of summing process. The interval between the summing amplifier and pre-amplifier cannot be negligible due to the difference in speed. The all pass filter helps to optimize the summing operation by delaying the pre-amplified noise in order to align the pre-amplified noise to the auxiliary input signal.

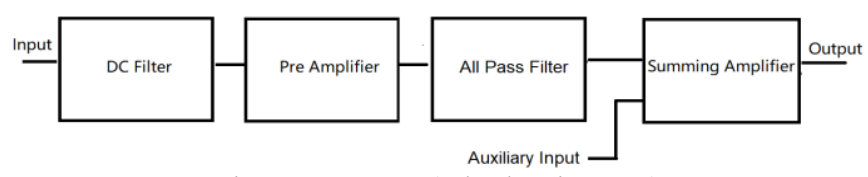

Figure 3. ANC (Block Diagram)

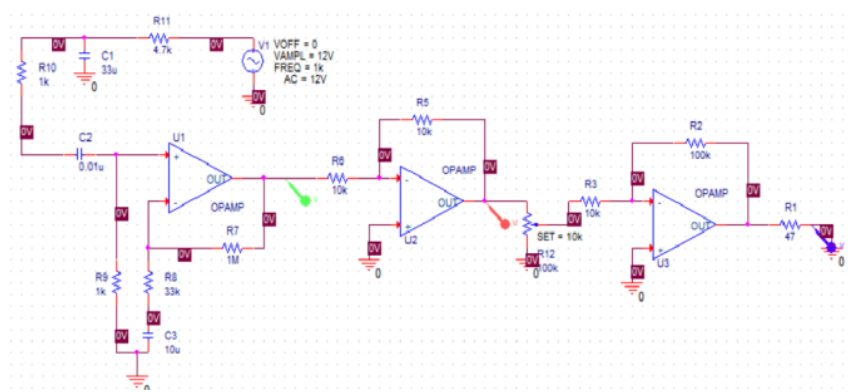

Figure 4. Schematic diagram of the ANC circuit.

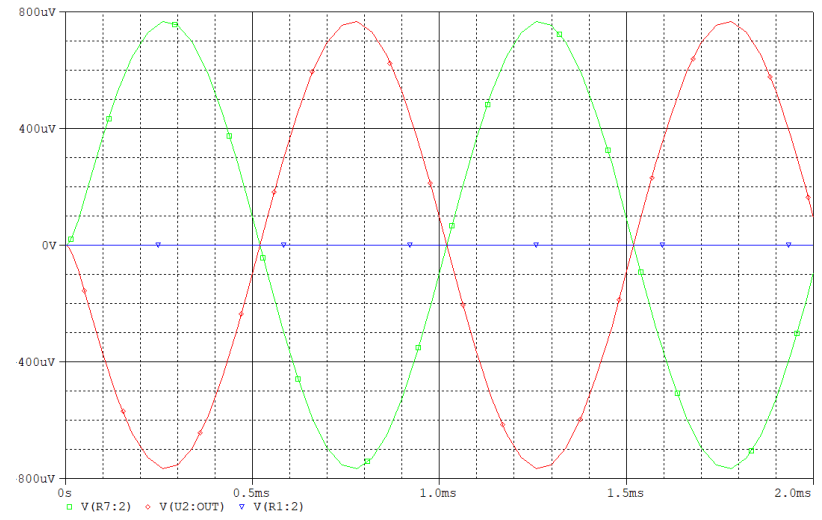

Figure 5. The simulation result using OrCAD Pspice.

The last stage is the summing amplifier. The auxiliary input superposition is formed with the combination of the auxiliary input and the amplified noise. The summation of the two signals is then inverted. A potentiometer is needed to fine tune the gain of the amplified signal to be matched with the amplitude of the sound signal. It is vital as only the suitable tuning of the gain will produce the ideal noise cancellation.

Despite the analog circuit, it is difficult to realize a device that can effectively cancel the noise as the commercial products that used the DSP to perform the complex computations in real time.

\section{EXPERIMENT EVALUATION}

\section{A. Experimental Testbed}

A sound level test is tested with different frequencies at different distance at different degree in a closed $440 \mathrm{~cm} \mathrm{x}$ $255 \mathrm{~cm} \times 265 \mathrm{~cm}$ room.

\section{B. Excitation of signals}

The signals with frequencies ranged from $100 \mathrm{~Hz}$ to $10 \mathrm{KHz}$ were used as noise source to create a diffuse sound field. The frequency signal was generated by Android app "Frequency Generator" and a 
Bluetooth speaker is used to play the sound signal. The frequency played was tested with Android app "Spectroid" for accuracy.

\section{Experimental Testbed}

The experimental testbed is shown in Figure 6 which consists of:-

- Android smartphones

- Smart Tools app as the sound level meter software

- Bluetooth Speaker

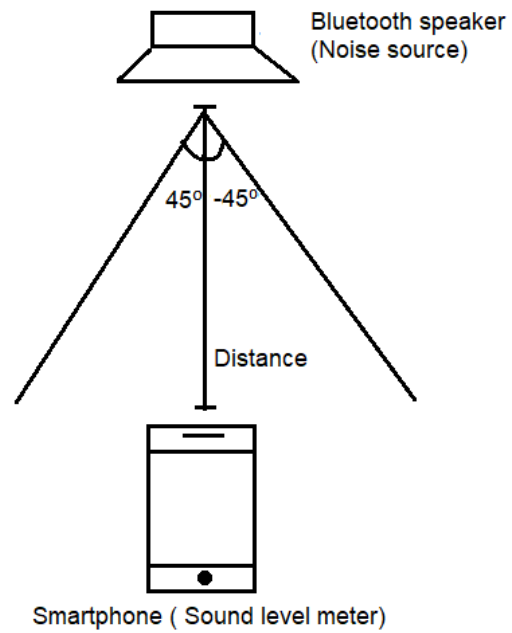

Figure 6. Measurement setup.

\section{Measurement Procedure}

As shown in Figure 6, the Bluetooth speaker is connected with another smartphone to generate a single frequency at one time. The sound level meter is used to measure the noise level. The distances between the meter and the noise signal source are increased in a step of $20 \mathrm{~cm}$ from $0 \mathrm{~cm}$ until $200 \mathrm{~cm}$. Each frequency will be measured with different distances and different angles, which are $45^{\circ}, 0^{\circ}$ and $-45^{\circ}$.

The distances between the noise source and sound level meter are measured using a measuring tape. The test is started with $0^{\mathrm{O}}$ measurement. A single frequency is played for 5 seconds and reading will be taken from the sound level meter. The reading is taken starting with $0 \mathrm{~cm}$ from the noise source and increased in a step of $20 \mathrm{~cm}$ each until $200 \mathrm{~cm}$. The $\pm 45^{\circ}$ measurements are conducted by using a protractor to measure the degree and the rope is used to align the respective degree with the noise source. The same procedures are carried out to measure the noise level under different angles.

\section{RESULT AND DISUSSION}

Figure 7 shows the noise level versus distance for different frequency at $45^{\circ}$. The highest noise level is $88 \mathrm{~dB}$ at $0 \mathrm{~cm}$ for $300 \mathrm{~Hz}$ whereas the lowest noise level found is $53 \mathrm{~dB}$ at $200 \mathrm{~cm}$ for $900 \mathrm{~Hz}$ and $160 \mathrm{~cm}$ for $1000 \mathrm{~Hz}$. As shown in Figure 8, the signal noise level measured corresponding to the distance for different frequency at $0^{\circ}$ is $87 \mathrm{~dB}$ at $0 \mathrm{~cm}$ for $300 \mathrm{~Hz}$ whereas the lowest noise level determined is $53 \mathrm{~dB}$ at $200 \mathrm{~cm}$ for $800 \mathrm{~Hz}$ and $1000 \mathrm{~Hz}$. Figure 9 shows the noise level versus distance for different frequency at $-45^{\circ}$. The highest noise level is $87 \mathrm{~dB}$ at $0 \mathrm{~cm}$ for $300 \mathrm{~Hz}$ whereas the lowest noise level is $52 \mathrm{~dB}$ at $200 \mathrm{~cm}$ for $1000 \mathrm{~Hz}$.

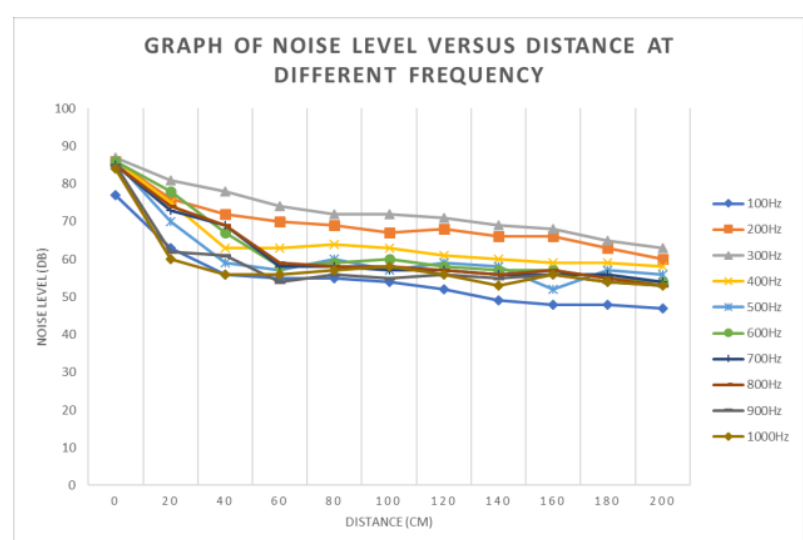

Figure 7. The noise level captured versus distance at different frequency $\left(45^{\circ}\right)$.

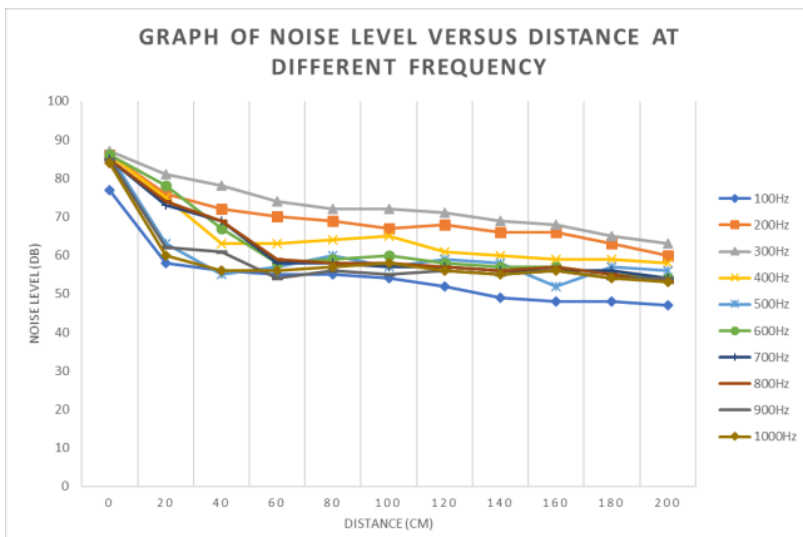

Figure 8 . The noise level captured versus distance at different frequency $\left(0^{\circ}\right)$

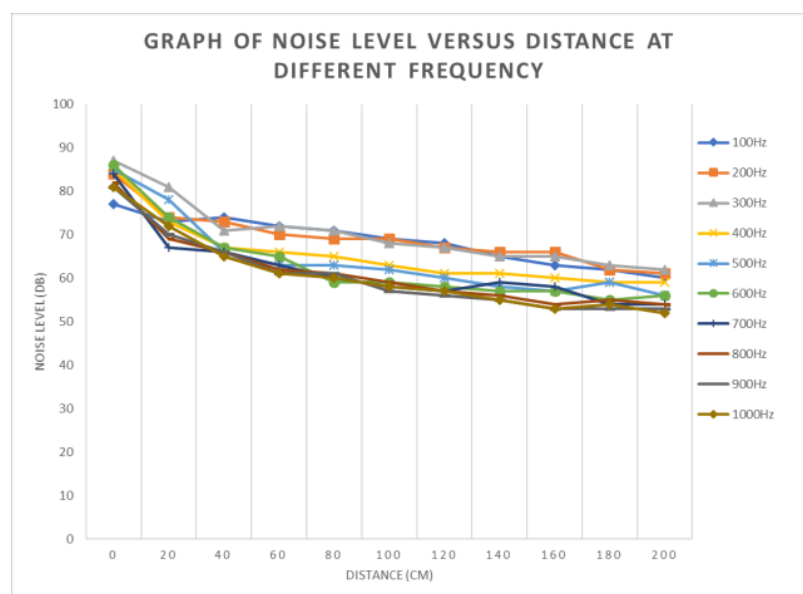

Figure 9. The noise level captured versus distance at different frequency $\left(-45^{\circ}\right)$.

From the extensive experimental results collected, it is found that the $100 \mathrm{~Hz}$ at $0 \mathrm{~cm}$ always have the lowest noise level compared to the same distance with different frequency. Besides, the experiments also achieve the highest noise level at $0 \mathrm{~cm}$ for $300 \mathrm{~Hz}$. They showed the similar trend 
regards the results. The biggest differences in noise level happened when the distance changed from $0 \mathrm{~cm}$ to $20 \mathrm{~cm}$. This is due to when the sound level meter is located next $(0 \mathrm{~cm})$ to the noise source, the microphone can receive the full sound wave of the frequency. When the distance increases to $20 \mathrm{~cm}$, there is air pressure that will cause compression and rarefaction of the sound wave travelled and caused the difference of magnitude up to $17 \mathrm{~dB}$. The magnitude of the noise level decreases as the distance increases regardless of the frequency generated.

\section{V.EXPERIMENTAL TEST FOR THE LOW COST ANALOG FEEDBACK ANC SYSTEM}

The excitation of signal used in this experiment is the broadband noise. The performance comparison of [7], [8] and [9] are carried out and all achieved the similar results for frequency ranged $250-700 \mathrm{~Hz}$. For the noise cancellation at $700-1500 \mathrm{~Hz},[7]$ outperformed the other works which can attenuate the noise up to $15 \mathrm{~dB}$. The proposed system can only attenuate $2-5 \mathrm{~dB}$ for both $250-700 \mathrm{~Hz}$ and $700-1500 \mathrm{~Hz}$, which is relatively low compared to the others. However, as it is a low-cost ANC circuit which does not use of any microcontroller or digital signal processor (DSP) and the cost to design the whole circuit is much lower than the systems that using DSP, which costs about USD $\$ 100 \sim 300$ [10], and is not affordable for the public. The power consumption of [7] is as low as $4.37 \mathrm{~mW}$ whereas the proposed design consumes $500 \mathrm{~mW}$, it still falls in the low power consumption range. However, the total cost to design the proposed system is only around USD \$10 which is an alternative and affordable choice with acceptable performance to implement the active noise cancellation functionality.

\section{CONCLUSION}

This paper explains the theoretical considerations on ANC system. The functionality of the analog feedback ANC system using the basic electronics components compensate the performance of noise cancellation with lower product cost. The low-cost ANC system proposed is affordable and it can be an alternative to adopt the ANC system. Besides, from the experimental results, it can be concluded that the magnitude of the noise level decreases as the distance increases regardless of the frequency generated.

\section{REFERENCES}

1. J. H. Lin, P. C. Li, S. T. Tang, P. T. Liu, and S. T. Young, "Industria wideband noise reduction for hearing aids using a headset with adaptive feedback active noise cancellation," Med. Biol. Eng. Comput., vol. 43, no. 6, Nov. 2005, pp. 739-745.

2. P. Lueg, "Process of silencing sound oscillations," U.S. Patent 2043 416, June 9, 1936

3. S.M. Kuo, D.R. Morgan, Active noise control systems, algorithms and dsp implementations, 3rd ed. New York, Wiley, 1995, pp. 470-596.

4. A. Tuomas, Erkki Heikkola, and Jari Toivanen, Active noise control in a stochastic domain based on a finite element model, University of Jyväskylä, 2009.

5. J.M. Sousa, C.A. Silva, and J.M. Costa, "Fuzzy active noise modeling and control of enclosures," IFAC Proceedings, vol. 35.1, 2002, pp. 235-240.

6. Kajikawa, Yoshinobu, W.S. Gan, and S.M. Kuo, "Recent advances on active noise control: open issues and innovative applications," APSIPA Transactions on Signal and Information Processing 1, 2012, pp. 3.
7. H.S. Vu, K.H. Chen, S.F. Sun, T.M. Fong, C.W. Hsu, and L. Wang, “A Power-Efficient Circuit Design of Feed-Forward FxLMS Active Noise Cancellation for In-ear Headphones," Feng Chia University, 2015.

8. M. Guldenschuh and R. Höldrich, "Prediction filter design for active noise cancellation headphone," IET Signal Process., vol. 7, no. 6, Aug. 2013, pp.497-504.

9. L. Zhang, L. Wu, and X. Qiu, "An intuitive approach for feedback active noise controller design," Applied Acoustics, vol. 74, no. 1, Jan. 2013, pp.160-168.

10. C.Y. Chang and S.T. Li, "Active Noise Control in Headsets by Using a Low-Cost Microcontroller," IEEE Transactions On Industrial Electronics, vol. 58, no. 5, May 2011, pp. 1936-1942.

\section{AUTHORS PROFILE}

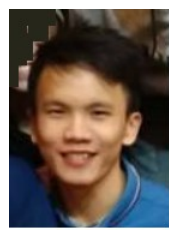

Soo Joon Yee received B.E degree in Electronics from Multimedia University, Malaysia in 2019. His research interests focus on the active noise cancellation and electronics circuit design.

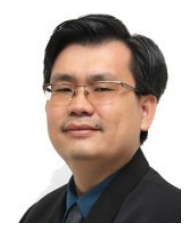

Pang Wai Leong received his Ph.D. degree in 2013. He is a Professional Engineer with Practising Certificate with Board of Engineers Malaysia and a corporate member with The Institution of Engineers Malaysia. His research interests include wireless communications, networking, VLSI, digital design and renewable energy. Since 2004, he has been with Multimedia University, Malaysia. Currently, he is the Programme Coordinator of the B.Eng. (Hons) Electronics under Faculty of Engineering, Multimedia University, Malaysia.

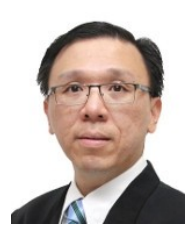

Chan Kah Yoong obtained his Ph.D. in Electrical Engineering from Jacobs University Bremen in Germany in year 2008. He is a Professional Engineer with Practising Certificate (PEPC) with BEM, a senior member with IEEE, and a corporate member with IEM. Ir. Dr. Chan research areas are in the micro and nanoelectronic device fabrications and characterizations Currently, he is an Associate Professor with Faculty of Engineering in Multimedia University, and serving as a chairperson for a Research Center under Faculty of Engineering of MMU - Centre for Advanced Devices and Systems (CADS). 